\title{
Narrative review of the influence of high-intensity interval training on adolescents' bone health: commentary and perspectives
}

\author{
Matias Noll ${ }^{1,2} \wedge$, Carolina Rodrigues Mendonça ${ }^{2} \wedge$, Ana Paula dos Santos Rodrigues ${ }^{3 \wedge}$, \\ Alexandre Aparecido de Almeida ${ }^{4}$, Priscilla Rayanne E Silva Noll ${ }^{1,5} \wedge$ \\ ${ }^{1}$ Instituto Federal Goiano (IF Goiano), Goiás, Brazil; ${ }^{2}$ Universidade Federal de Goiás (UFG), Goiás, Brazil; ${ }^{3}$ Superintendence of Health Surveillance, \\ Department of Goiás State, Goiás, Brazil; ${ }^{4}$ Instituto Federal de Educação, Ciência e Tecnologia do Tocantins, campus Araguatins (IFTO Araguatins), \\ Tocantins, Brazil; ${ }^{5}$ Departament de Obstetrícia e Ginecologia, Faculdade de Medicina (FMUSP), Universidade de São Paulo, São Paulo, Brazil \\ Contributions: (I) Conception and design: M Noll, CR Mendonça, PRES Noll; (II) Administrative support: M Noll, AP Rodrigues, PRES Noll; (III) \\ Provision of study materials or patients: M Noll, CR Mendonça, AP Rodrigues, de Almeida AA, PRES Noll; (IV) Collection and assembly of data: \\ M Noll, CR Mendonça, AP Rodrigues, de Almeida AA, PRES Noll; (V) Data analysis and interpretation: M Noll, CR Mendonça, AP Rodrigues, de \\ Almeida AA, PRES Noll; (VI) Manuscript writing: All authors; (VII) Final approval of manuscript: All authors. \\ Correspondence to: Matias Noll. Rua 17, Qd 53, Lt 10A, Centro, Rialma, GO, Brazil. Email: matias.noll@ifgoiano.edu.br.
}

\begin{abstract}
Although several benefits have been associated to high-intensity interval training (HIIT), there is a lack of clarity on the HIIT effects in adolescents, especially on bone health outcomes. To address this gap, our research aimed to perform a systematic review, which focus on the influence of HIIT on adolescents' bone health. Our search strategy was conducted on three databases (PubMed, SCOPUS, and Embase). For our review, we included articles with the following characteristics: (I) sample consisting of adolescents (10-19 years old), (II) HIIT interventions; and (III) assessment of bone health outcomes. Longitudinal and clinical trials studies with no language and year of publications restrictions were eligible to be included. A total of 63 eligible studies were identified. After removing the duplicates and screening the titles and abstracts, six articles remained to be read in full text. However, none of the articles met our criteria. Studies in which no article meets the eligibility criteria are also essential and need to be shared with the academic community because it may stimulate appropriate future investigations in this field. This lack in the review results highlights the need for further epidemiological research focusing on this topic, including high quality, large scale, and longitudinal studies, as well as randomized controlled trials to confirm or refute efficacy.
\end{abstract}

Keywords: Schoolchild; teenager; bone mineral content; bone mineral density (BMD); high-intensity interval training (HIIT)

Submitted Jun 28, 2020. Accepted for publication Oct 04, 2020.

doi: $10.21037 /$ tp-20-166

View this article at: http://dx.doi.org/10.21037/tp-20-166

\section{Introduction}

The development of bone mineral density (BMD) and content (BMC) during childhood and adolescence is substantial and may attenuate the incidence and effects of osteoporosis later in life (1). Recent study assessed 663 Danish students over 6 years from childhood to adolescence and found positive associations between the level of physical activity and bone health (2). Systematic reviews have shown that physical activity, as well as weight-

^ ORCID: Matias Noll, 0000-0002-1482-0718; Carolina Mendonça, 0000-0002-9902-8227; Ana Paula dos Santos Rodrigues, 0000-00020447-898X; Priscilla Rayanne e Silva Noll, 0000-0003-3715-1956. 
bearing exercises, enhance bone mineral accrual (3-6). According to current recommendations on physical activity for children and young people, vigorous-intensity activities are essential, "including those that strengthen muscle and bone, at least 3 times per week" (7). Both moderate and vigorous activities are indicated to reach wide health benefits, including bone health $(8,9)$, but the greatest benefit is provided by vigorous intensity physical activity (10-13), which is associated with the healthiest outcomes and the lowest risk of developing diseases later in life $(11,14,15)$.

Nowadays, high-intensity interval training (HIIT) is in the spotlight from both scientific and practical views. By definition, HIIT involves alternating short bursts of high intensity exercise with recovery periods or light exercise (16) and requires 'near maximal' efforts ( $\geq 80 \%$, maximal heart rate; or $\geq 80 \%$, maximal oxygen consumption) (17). Recent meta-review (18) analyzed 33 systematic reviews (child to adult) and found evidences suggesting that HIIT may improve anthropometric variables, exercise capacity, cardiorespiratory fitness, cardiovascular function, and anxiety and depression severity in both healthy individuals, as well as those with physical health disorders. Despite these health evidences support HIIT practice, only 5 reviews (19-23) from all 33 addressed children and adolescents, which reflects that the focus has been given to adult population.

Although several benefits have been associated to HIIT (18), to the best of our knowledge, there is a lack of clarity on the HIIT effects in adolescents, especially on bone health outcomes. So, here we aimed to perform a systematic review, which focus on the influence of HIIT on adolescents' bone health. We present the following article in accordance with the Narrative Review reporting checklist (available at http://dx.doi.org/10.21037/tp-20-166).

\section{Methods}

For the review, we included articles with the following characteristics: (I) sample consisting of adolescents (10-19 years old, as defined by the World Health Organization), (II) HIIT interventions; and (III) assessment of bone health outcomes (such as BMC or BMD). Longitudinal and clinical trials studies with no language and year of publications restrictions were eligible to be included. Studies with (I) incomplete data, (II) review articles, and (III) specific populations including athletes, hospitalized adolescents, diseased and disabled people were excluded.

Our search strategy was conducted on three databases
(PubMed, SCOPUS, and Embase). As the pattern, the following combinations of three sets of keywords were used in the Pubmed:

("adolescent”[MeSH Terms] OR “minors"[MeSH Terms] OR “adolescent”[Title/Abstract] OR “minors"[Title/ Abstract] OR "Youth"[Title/Abstract] OR "juvenile"[Title/ Abstract] OR "teen"[Title/Abstract] OR “teenager"[Title/ Abstract] OR "Childhood"[Title/Abstract] OR "adolescence"[Title/Abstract] OR "schoolchild"[Title/ Abstract] OR "Schoolchildren"[Title/Abstract] OR "young"[Title/Abstract])

AND

"bone density" [MeSH Terms] OR "bone health" [Title/ Abstract] OR "bone density" [Title/Abstract] OR "bone densities" [Title/Abstract] OR "bone mineral density" [Title/Abstract] OR "bone mineral densities" [Title/ Abstract] OR "bone mineral content" [Title/Abstract] OR "bone mineral contents" [Title/Abstract] OR "bone tissue" [Title/Abstract]) OR "bone and bones" [MeSH Terms] OR skeleton [MeSH Terms] OR skeleton [Title/Abstract] OR "bone mass" [Title/Abstract] OR "bone demineralization" [Title/Abstract] OR "bone development" [Title/Abstract] OR "densitometry" [MeSH Terms] OR "densitometry" [Title/Abstract] OR "bone architecture" OR "bone structure" OR "bone strength"

AND

("High-intensity training"[Title/Abstract] OR "Highintensity interval training"[Title/Abstract] OR "highintensity interval training" [MeSH Terms] OR "high intensity intermittent training"[Title/Abstract] OR "Repeated sprint training"[Title/Abstract] OR "interval training"[Title/Abstract] OR "intermittent training"[Title/ Abstract] OR "high intensity sprint"[Title/Abstract] OR "aerobic interval training"[Title/Abstract] OR "Highintensity interval training"[Title/Abstract] OR "highintensity interval exercise"[Title/Abstract] OR "highintensity interval exercise"[Title/Abstract] OR "highintensity intermittent exercise"[Title/Abstract] OR "highintensity intermittent exercise"[Title/Abstract] OR "high intensity intermittent training"[Title/Abstract] OR "high intensity intermittent training"[Title/Abstract] OR "HIIE"[Title/Abstract] OR "HIIT"[Title/Abstract]).

The same keywords groups have been used in the Scopus and Embase. The search was performed on 05 September 2020 by two independent researchers (MN and CRM), which also checked all the references in order to search for other potential articles. Then, the two reviewers ( $\mathrm{MN}$ and CRM) independently screened the titles and abstracts of 


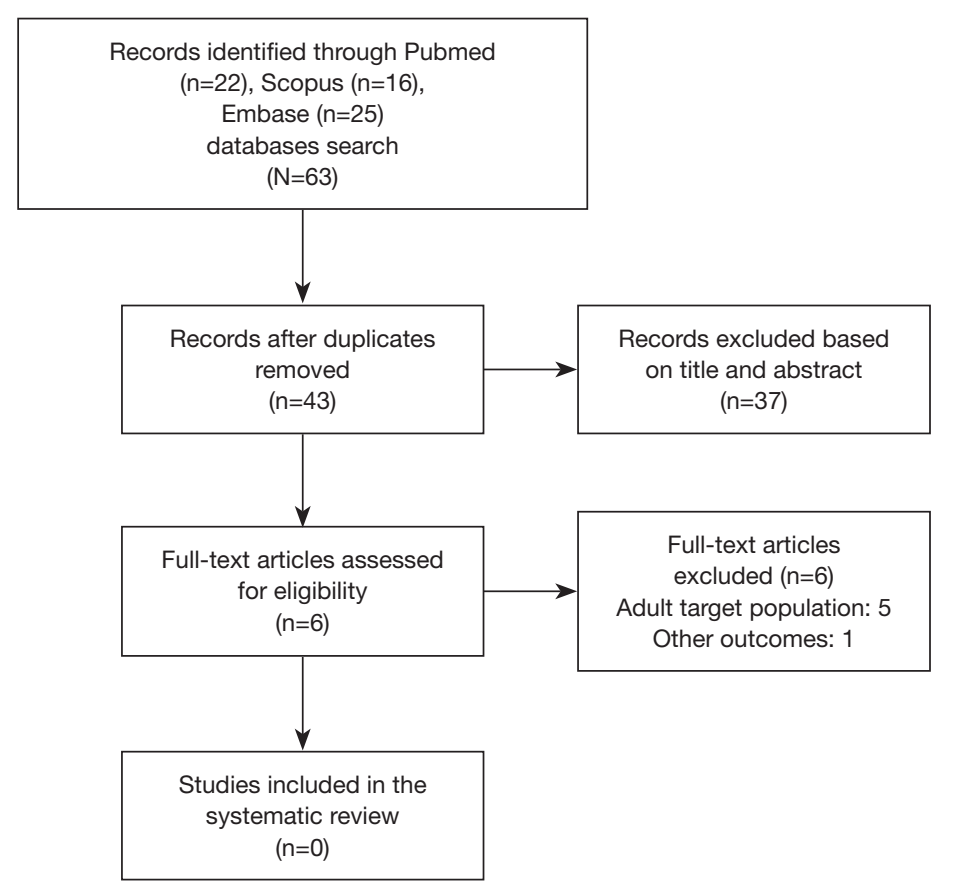

Figure 1 PRISMA flow diagram of the selection criteria for the study.

all articles identified in the literature search for inclusion. Disagreements regarding inclusion of articles were resolved by a third reviewer $(\mathrm{PN})$.

\section{Results}

A total of 63 eligible studies were identified (PubMed, 22 studies; Scopus, 16 studies; Embase, 25 studies). After removing the duplicates and screening the titles and abstracts, six articles remained to be read in full text (Figure 1). However, none of the articles achieved our criteria as all six studies were excluded because they were performed with adults.

\section{Discussion}

This lack of evidences on the influence of HIIT on adolescents' bone health emphasize the need for further observational studies, as well as randomized clinical trials to evaluate the effectiveness of HIIT on bone health. In this regard, we believe to be relevant sharing our findings in this Narrative Review, because this presented knowledge gap may motivate further studies in this thematic and help to develop this research line. According to Yaffe et al. (24), reviews in which no study meets the eligibility criteria are also essential and need to be shared with the academic community because it may stimulate appropriate future investigations in this field.

Given that (I) current recommendations suggest that vigorous intensity physical activity (10-13) for young people achieve large health benefits and reduce the risk of developing diseases later in life $(11,14,15)$, including bone health $(8,9)$, (II) HIIT may improve health-related outcomes $(19,21-23,25,26)$, and (III) the development of bone health during childhood and adolescence is substantial and may be an important protective factor for osteoporosis and fractures (1), it becomes essential to understand HIIT effects on bone health in adolescents, specially nowadays when this type of exercise is on spotlight. Briefly, recent studies with adults showed that HIIT can be an effective way to improve BMC and BMD (27), and regular powerbased training and competition in sprinting and jumping is associated with better maintenance of bone mass (28).

As previous evidences have suggested (18), HIIT may also improve anthropometric variables, cardiorespiratory and cardiovascular functions, and mental health. Studies mainly with adults have shown a good adherence rate of $\geq 80 \%$ and HIIT appears to be safe, with no acute injuries or serious cardiovascular events, similar to other active controls (18). Despite this positive evidences, HIIT adherence and adverse events should be carefully addressed 
in adolescent populations, for both sexes as well as different puberty stages. Further recommendations as type of movement, exercise duration and frequency, and rest intervals remain unclear due to the lack of studies currently available (23).

So, it is essential to understand more in-depth the HIIT effects on adolescents' bone health because it may provide a pragmatic complement to the Physical Education lessons (25) and underpin the formulation of national guidelines. Moreover, it may provide essential information for public health programs, as well as contribute to developing more specific and effective sports training programs, mainly directed to the school environment.

\section{Acknowledgments}

To the Instituto Federal Goiano [ifgoiano.edu.br] and the Child and Adolescent Health Research Group (GPSaCAwww.gpsaca.com.br) for the support.

\section{Footnote}

Reporting Checklist: The authors have completed the Narrative Review reporting checklist. Available at http:// dx.doi.org/10.21037/tp-20-166

Peer Review File: Available at http://dx.doi.org/10.21037/tp20-166

Conflicts of Interest: All authors have completed the ICMJE uniform disclosure form (available at http:// dx.doi.org/10.21037/tp-20-166). The authors declared no potential conflicts of interest with respect to the research, authorship and/or publication of this article.

Ethical Statement: The authors are accountable for all aspects of the work in ensuring that questions related to the accuracy or integrity of any part of the work are appropriately investigated and resolved.

Open Access Statement: This is an Open Access article distributed in accordance with the Creative Commons Attribution-NonCommercial-NoDerivs 4.0 International License (CC BY-NC-ND 4.0), which permits the noncommercial replication and distribution of the article with the strict proviso that no changes or edits are made and the original work is properly cited (including links to both the formal publication through the relevant DOI and the license). See: https://creativecommons.org/licenses/by-nc-nd/4.0/.

\section{References}

1. Min SK, Oh T, Kim SH, et al. Position Statement: Exercise Guidelines to Increase Peak Bone Mass in Adolescents. J Bone Metab 2019;26:225.

2. Rønne MS, Heidemann M, Lylloff L, et al. Bone Mass Development in Childhood and Its Association with Physical Activity and Vitamin D Levels. The CHAMPSStudy DK. Calcif Tissue Int 2019;104:1-13.

3. Behringer M, Gruetzner $S, M c C o u r t ~ M$, et al. Effects of Weight-Bearing Activities on Bone Mineral Content and Density in Children and Adolescents: A Meta-Analysis. J Bone Miner Res 2014;29:467-78.

4. Hind K, Burrows M. Weight-bearing exercise and bone mineral accrual in children and adolescents: A review of controlled trials. Bone 2007;40:14-27.

5. MacKelvie KJ. Is there a critical period for bone response to weight-bearing exercise in children and adolescents? a systematic review * Commentary. Br J Sports Med 2002;36:250-7.

6. Tan VP, Macdonald HM, Kim S, et al. Influence of Physical Activity on Bone Strength in Children and Adolescents: A Systematic Review and Narrative Synthesis. J Bone Miner Res 2014;29:2161-81.

7. World Health Organization (WHO). Global recommendations on physical activity for health. Switzerland: 2010.

8. Faienza MF, Lassandro G, Chiarito M, et al. How Physical Activity across the Lifespan Can Reduce the Impact of Bone Ageing: A Literature Review. Int J Environ Res Public Health 2020;17:1862.

9. Bland VL, Heatherington-Rauth M, Howe C, et al. Association of objectively measured physical activity and bone health in children and adolescents: a systematic review and narrative synthesis. Osteoporos Int 2020;31:1865-94.

10. Tarp J, Child A, White T, et al. Physical activity intensity, bout-duration, and cardiometabolic risk markers in children and adolescents. Int J Obes (Lond) 2018;42:1639-50.

11. García-Hermoso A, Ramírez-Campillo R, Izquierdo M. Is Muscular Fitness Associated with Future Health Benefits in Children and Adolescents? A Systematic Review and Meta-Analysis of Longitudinal Studies. Sports Med 2019;49:1079-94.

12. Farooq A, Martin A, Janssen X, et al. Longitudinal changes 
in moderate-to-vigorous-intensity physical activity in children and adolescents: A systematic review and metaanalysis. Obes Rev 2020;21:e12953.

13. Myers AM, Beam NW, Fakhoury JD. Resistance training for children and adolescents. Transl. Pediatr 2017;6:137-43.

14. Torres-Costoso A, López-Muñoz P, Martínez-Vizcaíno V, et al. Association Between Muscular Strength and Bone Health from Children to Young Adults: A Systematic Review and Meta-analysis. Sports Med 2020;50:1163-90.

15. Gibala MJ, Little JP. Physiological basis of brief vigorous exercise to improve health. J Physiol 2020;598:61-9.

16. Weston KS, Wisløff U, Coombes JS. High-intensity interval training in patients with lifestyle-induced cardiometabolic disease: a systematic review and metaanalysis. Br J Sports Med 2014;48:1227-34.

17. Viana RB, Naves JPA, Coswig VS, et al. Is interval training the magic bullet for fat loss? A systematic review and metaanalysis comparing moderate-intensity continuous training with high-intensity interval training (HIIT). Br J Sports Med 2019;53:655-64.

18. Martland R, Mondelli V, Gaughran F, et al. Can highintensity interval training improve physical and mental health outcomes? A meta-review of 33 systematic reviews across the lifespan. J Sports Sci 2020;38:430-69.

19. Costigan SA, Eather N, Plotnikoff RC, et al. Highintensity interval training for improving health-related fitness in adolescents: a systematic review and metaanalysis. Br J Sports Med 2015;49:1253-61.

20. Logan GRM, Harris N, Duncan S, et al. A Review of Adolescent High-Intensity Interval Training. Sports Med 2014;44:1071-85.

21. García-Hermoso A, Cerrillo-Urbina AJ, HerreraValenzuela $\mathrm{T}$, et al. Is high-intensity interval training more effective on improving cardiometabolic risk and aerobic capacity than other forms of exercise in overweight and

Cite this article as: Noll M, Mendonça CR, Rodrigues AP, de Almeida AA, Noll PRES. Narrative review of the influence of high-intensity interval training on adolescents' bone health: commentary and perspectives. Transl Pediatr 2021;10(1):160-164. doi: $10.21037 / \mathrm{tp}-20-166$ obese youth? A meta-analysis. Obes Rev 2016;17:531-40.

22. Engel FA, Ackermann A, Chtourou H, et al. HighIntensity Interval Training Performed by Young Athletes: A Systematic Review and Meta-Analysis. Front Physiol 2018;9:1012.

23. Eddolls WTB, McNarry MA, Stratton G, et al. HighIntensity Interval Training Interventions in Children and Adolescents: A Systematic Review. Sports Med 2017;47:2363-74.

24. Yaffe J, Montgomery P, Hopewell S, et al. Empty Reviews: A Description and Consideration of Cochrane Systematic Reviews with No Included Studies. PLoS One 2012;7:e36626.

25. Martin-Smith R, Cox A, Buchan DS, et al. High Intensity Interval Training (HIIT) Improves Cardiorespiratory Fitness (CRF) in Healthy, Overweight and Obese Adolescents: A Systematic Review and Meta-Analysis of Controlled Studies. Int J Environ Res Public Health 2020;17:2955.

26. Cao M, Quan M, Zhuang J. Effect of High-Intensity Interval Training versus Moderate-Intensity Continuous Training on Cardiorespiratory Fitness in Children and Adolescents: A Meta-Analysis. Int J Environ Res Public Health 2019;16:1533.

27. Brown EC, Hew-Butler T, Marks CRC, et al. The Impact of Different High-Intensity Interval Training Protocols on Body Composition and Physical Fitness in Healthy Young Adult Females. Biores Open Access 2018;7:177-85.

28. Ireland A, Mittag U, Degens H, et al. Greater maintenance of bone mineral content in male than female athletes and in sprinting and jumping than endurance athletes: a longitudinal study of bone strength in elite masters athletes. Arch Osteoporos 2020;15:87. 